\title{
EL OCIO EN LA PRIMERA INFANCIA DESDE UN ENFOQUE HUMANISTA EN TIEMPOS DE PANDEMIA
}

\author{
LEISURE IN EARLY CHILDHOOD FROM A HUMANISTIC \\ APPROACH IN TIMES OF PANDEMIC
}

\begin{abstract}
ב"
Carmen Andrés Viloria ${ }^{a}$, M. $^{a}$ Pilar Rodrigo Moriche $a^{a^{*}}$ y Paloma Valdivia Vizarreta ${ }^{b}$
\end{abstract}

Fechas de recepción y aceptación: 1 de septiembre de 2021 y 19 de noviembre de 2021

DOI: https://doi.org/10.46583/edetania_2021.60.947

Resumen: En la actualidad, una mirada a la vida cotidiana de la infancia nos permite observar la aceleración de los ritmos vitales, aspecto que se ha encontrado con tiempos de desconcierto durante la pandemia. Esta situación nos obliga a repensar, resituar y cuestionar la nueva realidad del tiempo libre y del ocio, y el aporte de estos al desarrollo personal y social en las primeras edades. A través de una revisión de la literatura, este trabajo pone énfasis en la importancia de cultivar de cero a seis años un ocio humanista. Para ello, se muestran elementos esenciales orientados a lograr un ocio voluntario y autotélico donde el juego es una de sus expresiones y la familia ostenta un papel clave para la educación de un ocio generador de salud, bienestar y calidad de vida. Se analizan también los tiempos y el ocio vivido durante la pandemia y la aceleración en el uso de las tecnologías en estas edades. A modo de conclusión, se destaca la relevancia y especificidad del ocio en la primera infancia; la necesidad de una dedicación familiar acorde a las inquietudes y deseos individuales; la potenciación de las figuras parentales en la construcción inicial del ocio; y se ofrecen unas orientaciones precisas según los tipos de ocio que aporta el enfoque humanista.

Palabras clave: primera infancia, ocio valioso, rol parental, ocio familiar, pandemia.

${ }^{a}$ Facultad de Educación. Universidad Autónoma de Madrid.

${ }^{*}$ Correspondencia: Universidad Autónoma de Madrid. Facultad de Educación. Ciudad Universitaria de Cantoblanco. 28049 Madrid. España.

E-mail: pilar.rodrigo@uam.es

${ }^{\mathrm{b}}$ Facultad de Educación. Universidad Autónoma de Barcelona. 
Abstract: Nowadays, a glance at the daily life of children allows us to see the acceleration of vital rhythms, an aspect that has met with times of bewilderment during the pandemic. This situation forces us to rethink, resituate and question the new reality of free time and leisure and their contribution to personal and social development in early childhood. Through a review of the literature, this paper emphasizes the importance of cultivating humanistic leisure from zero to six years. For this purpose, essential elements oriented to achieve a voluntary and autotelic leisure are shown, where play is one of its expressions and the family plays a key role in the education of a leisure that generates health, wellbeing and quality of life. The time and leisure experienced during the pandemic and the acceleration in the use of technologies at these ages are also analyzed. In conclusion, the relevance and specificity of leisure in early childhood is highlighted; the need for a family dedication according to individual concerns and desires; the empowerment of parental figures in the initial construction of leisure; and some precise guidelines are offered according to the types of leisure provided by the humanistic approach.

Keywords: early childhood, valuable leisure, parental role, family leisure, pandemic.

\section{INTRODUCCIÓN}

Los objetivos de la Agenda 2030 nos plantean promover sociedades justas, pacíficas e inclusivas que garanticen una vida sana y promuevan el bienestar para todas las personas en todas las edades. Nadie duda de que el ocio es un pilar importante del desarrollo humano, así como de su potencial educativo (Caride, 2014; Sarrate, 2008; WLRA, 1993). El derecho de la infancia al juego, al descanso, al esparcimiento y a las actividades recreativas y culturales está explícitamente ratificado en la Convención sobre los Derechos del Niño en 1989 (art. 31). Se consolida así el deber y la exigencia de resguardar plenamente a la infancia para que durante esta etapa vital crezcan, se desarrollen íntegramente, jueguen, descansen y aprendan (Unicef, 2018).

Si bien en la actualidad hay una mayor sensibilidad social a favor de una infancia saludable, cuidada y protegida, no resulta sencillo buscar ese equilibrio en el uso de los tiempos de juego, descanso y aprendizaje (Caballo, Gradaílle y Merelas, 2012). Existe un ocio nocivo o alternativas de ocio no adecuadas que constituyen una de las principales preocupaciones cuando hacemos referencia a las vivencias del ocio infantil en la sociedad actual (Valdemoros, Ponce de León, Sanz Arazuri y Caride, 2014). Un ocio más confuso si cabe ante la actual pandemia COVID-19, durante la cual el distanciamiento social ha reducido el juego, las relaciones sociales y las posibilidades de ocio en entornos educati- 
vos; ha aumentado la brecha educativa y se ha agudizado en algunos casos la vulnerabilidad infantil (Alonso, Sáenz de Jubera y Sanz, 2020; Unicef, 2014; Wang, Zhang, Zhao, Zhang y Jiang, 2020).

En general, son más numerosas las investigaciones que se han llevado a cabo sobre juventud, adolescencia e infancia y ocio (De Valenzuela, Martínez, y Escobar, 2021; Díaz-Herrera, Salcines-Talledo, y González-Fernández, 2021; Valdemoros et al., 2014; Sanz, Sáenz de Jubera y Cano, 2018), y menos las que inciden exclusivamente en la primera infancia (Ferreiro-Núñez, 2016), aunque comienza a existir la necesidad de analizar el ocio en esta etapa (Azagra y Giménez, 2018). Esta situación hace que aún haga falta indagar sobre el ocio durante los seis primeros años de vida y el papel parental para salvaguardar y fomentar una educación del ocio frente a las numerosas presiones que ejerce la vida moderna.

Una de las principales limitaciones que se encuentran en esta etapa es la falta de toma de consciencia de sus posibilidades participativas y de interacción, aspecto que deriva en ocasiones en su invisibilidad (Palacios y Castañeda, 2011). La finalidad de este trabajo es repensar los tiempos sociales y el valor del ocio como generador de salud, bienestar y calidad de vida en la primera infancia, y proyectar hacia una educación de ocio humanista que parta de sus intereses y necesidades individuales.

\section{JUEGO Y OCIO EN LA PRIMERA INFANCIA}

Es preciso comprender el concepto de ocio y las características que lo definen, pues de acuerdo con ello tendrá lugar -o no- aquello que consideremos como ocio saludable, generador de bienestar y calidad de vida para el desarrollo en estas edades. En este sentido, diferentes investigaciones que se han interesado en el ocio consideran necesario diferenciar los términos ocio y tiempo libre, puesto que disponer de tiempo libre no equivale a tener una vivencia de ocio (Aristegui y Silvestre, 2012; Stebbins, 2012). La clave para entender la experiencia de ocio es la persona que la experimenta (Cuenca, 2014).

El tiempo, en cuanto tiempo social, es considerado algo objetivo, medible y cuantificable; pero el ocio, como vivencia humana, se conecta necesariamente a una experiencia personal y subjetiva en la que resulta difícil cuantificar los 
momentos y efectos del proceso (Caballo, 2009; Cuenca, 2004; Gambara, Botella y Gempp, 2002). Aunque es cierto que en la infancia hay menos obligaciones que en la edad adulta y, en consecuencia, los niños cuentan con mayor tiempo libre, el hecho de considerar la experiencia de ocio como una percepción subjetiva nos lleva a determinar la percepción que se tiene en esta etapa.

El concepto y la percepción del tiempo se aprende y se interioriza en la infancia, al igual que ocurre con la percepción sobre el paso de este (Alonso et al., 2020; Sarrate, 2008). Esta percepción va a depender de cada niño. La investigación realizada por Caballo (2009) tiene en consideración las inquietudes y los deseos de niños y niñas respecto a su tiempo libre y encuentra que lo que más desean es tener tiempo para jugar, es decir, tiempo no estructurado, no pautado, tiempo autónomo. Por otra parte, se consideran como elementos esenciales del ocio la satisfacción, la creatividad, la diversión, la libertad, el crecimiento y el desarrollo personal (Cuenca, 2014; Ferreiro-Núñez, 2016).

El juego como expresión del ocio es una dimensión fundamental y vital del placer de la infancia, así como un componente indispensable del desarrollo físico, social, cognitivo y emocional (Aucouturier, 2018; Berrios, Lazcano, Madariaga, 2017). Es una vivencia que se inserta en el mundo de las emociones, de ahí la importancia de cultivarlo desde el afecto. En la infancia el ocio necesita conectar con la ilusión y con la curiosidad porque lo que se aprende está influido y organizado por las emociones (Mora, 2013). Va a ser fundamental que la vivencia de ocio les proporcione un clima emocional apropiado y se promuevan experiencias emocionales positivas, sin estrés, en las que haya una sensación de seguridad y relajación, sintiendo que pueden disfrutar. Se trata de proporcionar experiencias personales que conecten con sus intereses, necesidades y deseos.

Durante la situación de pandemia, sin los espacios de esparcimiento habituales, no solo se ha limitado la actividad física sino que también ha faltado la identificación y puesta en práctica de diferentes roles sociales y el desarrollo de un lenguaje más sofisticado e imaginativo. Para las familias ha sido todo un desafío reconfigurar y reinventar los tiempos y espacios en casa para jugar y aprender a través de juegos sensoriales y de manipulación para estimular los sentidos; juegos físicos o motores para promover un estilo de vida activo; juegos dramáticos o de simulación para estimular la imaginación y la creatividad; o el descarte y posterior dosificación del juego al aire libre. 
En este proceso de reinventarnos también han surgido soluciones desde las tecnologías. La investigación internacional ha comenzado a considerar el potencial del chat de vídeo para apoyar la interacción familiar intergeneracional. Este aspecto ha facilitado, por un lado, las relaciones por situaciones de confinamiento o lejanía geográfica; y, por otro, la contribución en estas situaciones al refuerzo del desarrollo cognitivo y socioemocional infantil (Zuhro, 2021). En comparación con una llamada telefónica, el chat de vídeo puede ser particularmente bueno para construir la cercanía familiar a través de la conversación y las propuestas lúdicas, pues abundan los gestos y las miradas compartidas, permite la comunicación mostrando objetos o juguetes, se les puede leer un libro y desarrollar propuestas para el juego. Así lo demuestran las investigaciones de videochat entre niños y niñas de 0 a 3 y sus padres/ madres en cárceles (Skora y Poehlmann-Tynan, 2020) o con abuelos/abuelas (Strouse et al. 2021).

El ocio debe contribuir al bienestar y la satisfacción y constituir un requisito indispensable para proporcionar calidad de vida en la infancia. Una buena educación del ocio no depende solamente de la cantidad de actividades, sino de la calidad, que sean actividades óptimas para las necesidades evolutivas.

\section{Papel de las familias en las PRIMERAS EXPERIENCIAS DE OCIO}

Si la vida buena se define por la riqueza de experiencias, como afirma Dávila (2016), hay que estar pendientes de las experiencias de ocio que se vivencian en los primeros años de vida. En edades tempranas, de 0 a 6 años, las necesidades y experiencias de ocio se suelen satisfacer en la familia y mediante esta. De ahí la importancia de tener en cuenta el rol que adquiere la familia en la experiencia educativa del ocio de sus hijos e hijas y su influencia. El ocio en familia es algo muy deseable que suceda como un punto de partida, ya que se fortalecen los vínculos, las relaciones, la autoestima; asimismo, se gana estabilidad y es un medio de formación y de encuentro para las familias (Bofarull, 2005; McCabe, 2015).

Son los factores relacionados con el funcionamiento interno familiar los verdaderos condicionantes en la construcción del ocio (Valdemoros et al., 2014). Es una educación que se inicia en la primera infancia, pero no es una 
tarea sencilla, ya que implica una reflexión desde la parentalidad positiva sobre las necesidades y la distribución de los tiempos sociales infantiles (Berrios et al., 2017; Rodrigo, 2015). Es muy importante acertar en la forma de educar (Andrés y Fernández, 2016).

Es fundamental que padres y madres durante la infancia de sus hijos puedan organizar un ocio educativo, estimulante y a la vez formativo que ayude a vivenciar experiencias de ocio de manera saludable. Bofarull (2005) señala la necesidad de un ocio familiar funcional que permita ocasiones de encuentro y de diversión en familia como una forma de transmitir oportunidades educativas y también de descanso y autorrealización. Este ocio funcional familiar combina ocio de diversión -aquel que permite el descanso, el cambio de actividad y el entretenimiento, y no exige aprendizaje, como ir al cine, comer juntos, ver la $\mathrm{TV}$, ir a parques temáticos, etc.- con ocio de satisfacción - un ocio que va más allá y busca la realización personal y familiar de cada miembro, y que necesita procesos de aprendizaje de actividades motrices, deportivas, culturales, etc.-Dentro de esta cotidianidad familiar se incluye también la influencia positiva de la abuelidad en el ocio (Alonso et al., 2020), capaz de incrementar competencias y habilidades en el desarrollo emocional, cognitivo, social, emocional y físico en la infancia (Berrios et al., 2017).

El ocio familiar funcional es un factor de protección en estas edades, pero hay que reconocer que exige esfuerzo y que, en ocasiones, la visión parental sobre el significado del ocio puede ser distinta (Leyra y Bárcenas, 2014). Si las familias se implican, enseñan a entretenerse y a jugar autónomamente; proporcionan ideas y oportunidades lúdicas y predeportivas; los llevan a la naturaleza, y los acompañan de forma activa y autónoma; los niños y las niñas construyen un ocio saludable (Bofarull, 2005).

Por otro lado, es preciso puntualizar el problema de la asincronía en los tiempos de ocio en la infancia y en la vivencia del ocio no compartido en el entorno familiar. Junto a la preocupación y la inquietud en torno a los horarios laborales de las familias también aparece la falta de tiempo libre para vivir momentos de familia, los cambios en las dinámicas familiares y las pautas de crianza y socialización (Andrés y Fernández, 2016; Barudy y Dantagnan, 2009; Sanz, 2014).

Si pensamos en la mercantilización y proliferación de las actividades extraescolares como ocupación del ocio en la infancia (Fraguela, Lorenzo y Vare- 
la, 2011; Morán de Castro, 2009), aparece en ocasiones un ocio no controlado de cerca por la familia, sino por el centro educativo. De este modo, el entorno escolar se convierte en uno de los principales agentes de la educación del ocio junto a las familias (Dávila, 2016).

En algunas ocasiones surge como una opción para ampliar el horario escolar y adaptarlo a la jornada de trabajo, permitiendo así la conciliación de la vida familiar y laboral; en otras ocasiones, responde a una elección voluntaria familiar que busca la vivencia de momentos de satisfacción y posibilitar prácticas beneficiosas para su desarrollo; o tal vez, como se ha señalado, a pesar de la presencia muy notable de actividades extraescolares en la agenda diaria de la infancia, se constituyen como un mal menor al que recurrir cuando las familias no pueden o no saben ocuparse de los tiempos de crianza (Caballo, 2009).

Cuando nos encontramos con esta profesionalización del tiempo libre, esta productividad del ocio durante la infancia, la consecuencia puede ser que cada vez sea más difícil que disfruten del ocio que enriquece; que nos encontremos ante un ocio que no responda a los gustos e intereses personales y se impongan como actividades obligatorias.

A pesar de existir muchas actividades de ocio interesantes, enriquecedoras y significativas para favorecer el desarrollo de la primera infancia, en ocasiones, es un ocio infantil sobreestimulado que responde a los desajustes y las asincronías que sus figuras parentales arrastran cotidianamente en sus modos de vida, lo que produce desequilibrios en sus procesos de desarrollo personal y social (Morán de Castro, 2009).

Existe la idea de que experimentar sentimientos de aburrimiento no es bueno, pero hay un punto del aburrimiento que es saludable, y no hay necesidad de estar llenando todo el tiempo libre. En ese aburrimiento es cuando les puede surgir cosas sumamente creativas, si es que se les da la oportunidad de aburrirse, por supuesto. Quizás, como nos dice Lecuyer (2012), apenas les dejemos tiempo y espacio para el asombro.

A lo anterior se añade la crisis por la COVID-19, que ha traído desafíos sin precedentes para la infancia. Se han producido cambios en sus rutinas y han convivido con personas adultas con estrés por la incertidumbre, el trabajo, la dinámica familiar, la conciliación y su tiempo libre; y donde las familias también desempeñaron el apoyo más importante para su aprendizaje: les contaban 
cuentos $(80 \%)$, cantaban canciones $(62 \%)$ y trabajaban con ellos números y letras al menos tres veces por semana (61\%) (Weiland et al., 2021).

Todos los aspectos señalados afectan a la toma de decisiones sobre el ocio en la crianza (Berrios et al., 2017; Valdemoros et al., 2014). Las familias son las que dan sentido a las experiencias infantiles y son esenciales para amortiguar la sensación de estrés, y ayudarlos a manejar sus sentimientos (Andrés y Fernández, 2016).

\section{ApORTES DE UN OCIO HUMANISTA}

El ocio humanista se entiende como un ocio de liberación que conlleva la voluntad de querer hacer algo, autotélico, con un fin en sí mismo, y que requiere formación para evitar el ocio nocivo (Kriekemans, 1973). Un ocio entendido como derecho, ámbito de libertad y recurso de desarrollo personal y social para la mejora de la calidad de vida (Cuenca, 2000).

Este proceso continuo de aprendizaje influye directamente en el desarrollo de actitudes, valores, conocimientos y habilidades. Se establece, por tanto, una estrecha reciprocidad entre aprendizaje y desarrollo, lo que requiere de una sucesiva adaptación a cada edad y a las circunstancias personales. La clave no solo es pensar que el ocio es óptimo para el desarrollo infantil, sino que este tiene que estar en armonía con sus intereses y necesidades vitales.

Por otro lado, se apunta que el ocio humanista promueve una buena salud, bienestar y calidad de vida al ofrecer distintas oportunidades que permiten a individuos y grupos seleccionar actividades, intereses y preferencias (Fraguela et al., 2011). Un bienestar que no es ajeno al bienestar de la familia (Rodrigo, 2015).

Existe también una clara evidencia de que las experiencias y condiciones biopsicosociales en los primeros años de vida tienen un impacto crucial sobre la salud física y psíquica (Andrés y Fernández, 2016). Ideas que confirman que las experiencias de ocio que generan estrés y presión son generadoras de hábitos poco saludables, cuyas consecuencias pueden llegar a alcanzar especial vulnerabilidad en la infancia (Mora, 2013; Morán de Castro, 2009).

No existe un único modo de ocio, sino muchos y posibles. Las personas adultas tienen la responsabilidad de orientar, guiar y diferenciar el tipo de ocio 
adecuado en la infancia en función de tres aspectos: la edad, la capacidad de simbolización y las condiciones en las que se realiza el ocio.

Desde el prisma humanista del ocio, la educación de este en la primera infancia se orienta hacia:

- Un ocio activo. Es un ocio elegido, deseado y querido, un ocio en el que nos implicamos. Se produce a medida que se crece y evoluciona en las distintas etapas del desarrollo. Para cada individuo la experiencia de ocio es distinta. En la infancia hay un interés innato por explorar, curiosear y estar disponibles para inventar, crear y jugar (Piaget, 2000) que es consecuencia del proceso individual de madurez cerebral y corporal siendo además el motor del aprendizaje (Mora, 2013). Este ocio activo debe contribuir al bienestar de la infancia (Rodrigo-Moriche y Vallejo, 2015) y las familias ostentan esta responsabilidad.

- Un ocio sustancial. Son prácticas de ocio arraigadas que se llevan a cabo de un modo reiterado y se cargan de sustancia, de contenido, de significación. El primer paso en el proceso evolutivo en edades tempranas es establecer vínculos afectivos y emocionales con las personas de su entorno. Las familias deben modelar y fomentar un ocio humanista tomando decisiones respecto a la ampliación del mundo social de sus hijos, acompañarlos en una mayor exploración de la realidad social que los rodea, permitirles una creciente participación en las actividades y experiencias de la familia y en el mundo de los iguales (Rodrigo, 2015). Es fundamental fomentar espacios y encuentros de este tipo, ya que mediante estas interacciones en la infancia se aprende a ser grandes observadores de otros intereses y perspectivas. Cuando juegan con sus iguales tienen que escuchar, esperar, desarrollar la empatía, aprender a perder, hacerse escuchar, controlar la ira, aprender a negociar, establecer turnos y reglas y aprender a defenderse de una forma adecuada.

- Un ocio lúdico o creativo. Es un ocio automotivado, consciente, activo y complejo. Se caracteriza por la vivencia de experiencias relacionadas con el juego y las distintas formas de entenderlo, ya sea de carácter físico o mental. El juego en la infancia se activa como un mecanismo natural donde se transforma lo real en función de sus deseos, lo dota de afecto, revive todos sus placeres o todos sus conflictos, pero sobre todo crea un mundo de fantasía. Mediante el juego se desarrolla el lenguaje, la simbolización, 
la psicomotricidad y la madurez emocional (Aucouturier, 2018; Piaget, 2000; Pikler, 1984), y potencia el desarrollo integral en todas sus facetas (motoras, cognitivas, sociales, etc.). De manera concreta, el juego heurístico y el juego simbólico en estas edades constituyen una actividad real del pensamiento y ese proceso ya es una experiencia emocional.

Las oportunidades frecuentes de juego permiten expresar emociones y sentimientos; mejorar las relaciones sociales (Delgado, 2012); tener más capacidad de llevarse bien con otros; $y$, en definitiva, crecer más sanos y felices (Burdette y Whitaker, 2005; Romero y Gómez, 2000).

- Un ocio cultural y festivo. Es un ocio motivado por el disfrute de las distintas expresiones culturales y llevado a cabo a través del conocimiento. Es un ocio eminentemente formativo e implica una vivencia subjetiva (Águila, 2007). La lectura infantil, contar cuentos y compartir historias son experiencias fundamentales y tienen un gran atractivo en estas edades. Representan una forma de ordenar el mundo y dar sentido a los acontecimientos de la vida (Braid y Finch, 2015). Al escuchar, se practican habilidades del pensamiento tan importantes como predecir, analizar, relacionar e inferir (Sanz, 2014).

Desde el punto de vista comunitario, cada vez se ofrecen más posibilidades de actividades de ocio familiar (Azagra y Giménez, 2018) en las que se puede asistir a conciertos, cantar en familia, ir a espectáculos de danza, a teatros, a museos, etc.

- Un ocio ambiental-ecológico. Se trata de la vivencia de experiencias satisfactorias motivadas por el contexto en el sentido de "estar" en un lugar y/o un ambiente. Tal y como Freire (2011) expresa, la naturaleza y la vida al aire libre promueven un desarrollo holístico en la infancia y potencian la autonomía, la responsabilidad, el autoconocimiento y la autoconfianza. La naturaleza les concede un sentimiento global de pertenencia al mundo, promueve sus capacidades sociales y comunicativas a la vez que reduce la probabilidad de enfermedades, aumenta la creatividad y multiplica las facetas del juego.

Más allá de todos los beneficios sobre la salud y el equilibrio emocional, también encuentran en la naturaleza situaciones, contextos y problemas reales en los que desenvolverse y poner en práctica sus estrategias y habilidades investigando, experimentando, equivocándose, cooperando 
y aprendiendo innumerables enseñanzas (Corraliza y Collado, 2011; Mora, 2013; Wells, 2000).

El entorno donde se vive condiciona y, por eso, la naturaleza puede ser considerada como una referencia por ser el lugar donde viven; o en otros casos, pueden tener los síntomas del llamado trastorno por déficit de naturaleza (Louv, 2008).

- Un ocio deportivo. La motivación principal que incita a realizar la acción de una práctica deportiva es porque nos gusta. Durante los primeros años de vida, partir de una perspectiva de libertad del movimiento y estimular el desarrollo motor de modo espontáneo y autónomo favorecerán la motivación intrínseca de explorar mediante el juego sus habilidades motoras (Pikler, 1984) y facilitar el disfrute del movimiento para posteriormente establecer conexión con la experiencia deportiva.

La OMS ha publicado la guía Physical activity, sedentary behaviour and sleep (2019) sobre niños menores de 5 años. Al referirse a actividad física, recomienda ejercicio físico de intensidad moderada a vigorosa cada día a través de juegos, deportes, transporte, recreación y educación física en el contexto de la familia, la escuela y las actividades comunitarias. Define también la importancia del juego interactivo con la familia para el aprendizaje cognitivo y motor. En contraste, el comportamiento sedentario se refiere a estar en el cochecito, en la cuna, y también al tiempo frente a las pantallas.

- Un ocio solidario. Se refiere a una experiencia en la que las vivencias satisfactorias están motivadas por el hecho de ayudar "a la otra persona”. Esta característica esencial determina que la educación en valores sea el núcleo de las experiencias solidarias y que estas encuentren su razón de ser en los valores. La educación en valores acerca a la infancia a este tipo de ocio. Una de las principales funciones de la familia es la transmisión de valores sociales (Marín, Quintero y Rivera, 2019) y los cuentos se presentan como una herramienta esencial (Marín-Díaz y Sánchez-Cuenca, 2015).

Si bien el ocio humanista se caracteriza, entre otros, por la libre elección de la persona, aún hay que determinar cuánto de libre es el ocio en la primera 
infancia y qué influencia ejercen las posibilidades que ofrece a los niños y niñas su entorno para el desarrollo de este ocio en etapas posteriores.

\section{LA PANDEMIA Y EL OCIO TECNOLÓGICO EN LA PRIMERA INFANCIA}

El acontecimiento imprevisto de la pandemia por la COVID-19 ha alterado profundamente las interacciones familiares. Ha sido una situación de cambios significativos en las dinámicas sociales infantiles. Las familias han tenido que compatibilizar con sus trabajos el apoyo en la educación en el hogar en ausencia de los sistemas de apoyo social externos. Se han cancelado y se han reestructurado las actividades de ocio, tanto en el entorno escolar como en el tiempo de ocio compartido en familia, y en muchas ocasiones no hay espacios adecuados donde desarrollar el juego y el deporte (Wang et al., 2020). Existen ya estudios que señalan una relación entre el aislamiento y las restricciones de contacto físico en el esfuerzo por reducir la transmisión de COVID-19 y los primeros signos sutiles de problemas de salud relacionados con el aumento de la obesidad infantil por actividad física inadecuada. La repercusión que sobre el desarrollo en estas edades puede suponer la pandemia actual, en general, ha evidenciado la falta de actividades físicas al aire libre, mayor tiempo frente a las pantallas digitales, situaciones de mayor estrés para gestionar la educación en casa y más dificultades alimentarias por el consumo de alimentos más procesados y ricos en calorías. Muchas de las consecuencias referidas van a depender de la edad, la raza y el origen étnico, así como el seguro médico y los ingresos familiares (Jenssen et al., 2021).

También se ha señalado que el confinamiento en el hogar durante la pandemia ha permitido aumentar el tiempo dedicado al juego tecnológico, con una elevada disponibilidad de dispositivos de pantalla táctil para estas edades. Los estudios introducen la reflexión sobre el impacto de la tecnología durante la pandemia de acuerdo con las culturas, sus prácticas y percepciones (Dardanou et al., 2020). En este sentido, los estudios identifican que es la familia la que juega un papel muy importante en la creación de hábitos respecto al tiempo frente a las pantallas. Sus actitudes, creencias, modelos y estilo de crianza dejan huella en el desarrollo de sus hijos e hijas (Konok, Bunford y Miklósi, 2020; Yogman et al., 2018). 
Según las recomendaciones de los profesionales de la American Academy of Pediatrics (Council on Communications and Media, 2016), se adopta el criterio de iniciar paulatinamente el uso de tecnología en la infancia partiendo de la supervisión y orientación adulta. Estas recomendaciones señalan que menores de 2 años solo pueden exponerse a videollamadas cortas. Un uso excesivo de la tecnología se ha asociado con una serie de riesgos de salud en estas edades, relacionados con la reducción del sueño, el aumento de la obesidad, mala adaptación social y retrasos socioemocionales y del lenguaje. Sin embargo, los datos indican que más de un tercio de niños y niñas entre 1 y 2 años ya ha comenzado a usar dispositivos móviles (Konok et al., 2020).

El informe The common sense census: media use by kids age zero to eight (2017) señala que antes de la pandemia la incidencia del uso de la pantalla hasta los 4 años oscilaba entre 42 minutos (de 0 hasta 23 meses) hasta 2 horas y 39 minutos (de 2 a 4 años) al día, y la mayor parte de este tiempo frente a la pantalla consistía en ver vídeos (72 \%). Según Kotrla, Varga y Jelovčić (2021), durante el confinamiento por la COVID-19 se incrementó el uso de los dispositivos entre toda la familia, ya sea por trabajo, por estudios, por ocio o para comunicarnos. Las familias estimaron que entre un tercio y casi la mitad de niños y niñas, en todos los grupos de edad, pasaron más tiempo usando los dispositivos tecnológicos, para comunicarse con amistades y familiares, como entretenimiento o para pasar el tiempo y relajarse, que antes del confinamiento por la emergencia.

En este sentido, la investigación de Cartanyà-Hueso et al. (2021) sostiene que tres de cada cuatro niños y niñas, de 12 a 47 meses, estuvieron expuestos más tiempo que antes a teléfonos inteligentes y tabletas durante las comidas $\mathrm{y}$ antes de acostarse.

Dada la plasticidad neuronal significativa en estas edades, desde diferentes estudios se pretende conocer si el uso excesivo de la tecnología afecta al desarrollo del cerebro, observando cuáles serían las consecuencias cognitivas y para el aprendizaje de esos posibles cambios. Las investigaciones ofrecen resultados mixtos o contradictorios (Da Rosa Piccolo, Weisleder, Mendelsohn, 2020; Dardanou et al., 2020; Hawkey, 2019; Kalnina y Kalnins, 2020). Aunque no se comprende completamente cuál es el efecto en este periodo crítico, se relaciona la falta de atención a corto plazo con preferencias por gratificaciones 
inmediatas; y el uso prolongado de teléfonos inteligentes para el ocio está vinculado negativamente con la calidad del sueño (Kotrla et al., 2021).

Los estilos de crianza digital influyen tanto en el tiempo de uso de los dispositivos móviles de pantalla táctil como en el uso en sí mismo (Konok et al., 2020). Las familias juegan un papel importante en la creación de un ambiente hogareño y promueven ciertos comportamientos y roles que definen hábitos y estilos de vida, por ejemplo, en relación con la cantidad de actividad física, el comportamiento sedentario o el tiempo frente a la pantalla.

\section{Conclusiones}

Este trabajo ha puesto la mirada en la educación del ocio en la primera infancia, demostrando su relevancia y especificidad, y la necesidad de una especial dedicación desde el entorno familiar. Desde un punto de vista humanista, las experiencias de ocio centradas en quien lo experimenta nos conducen a tener en cuenta las inquietudes y los deseos individuales en la infancia. En este sentido, el juego, como expresión de ocio, se presenta como la mejor manera de enseñar. En concreto, el juego social y no estructurado realizado en familia promueve la función ejecutiva y el cerebro prosocial, tal y como han apuntado algunos estudios (Yogman et al., 2018).

La situación de pandemia nos ha permitido ver lo mejor y lo peor de las experiencias que vive la infancia en su tiempo libre. Ha pasado el tiempo y nos ha mostrado la plasticidad y flexibilidad para reimaginar e inventar nuevas posibilidades con las que conceptualizar y percibir el tiempo desde casa; ha acelerado el uso de las tecnologías en edades cada vez más tempranas; y nos ha señalado de nuevo la importancia sobre el uso del tiempo y la valoración de los contenidos y el contexto en el que se utilizan las tecnologías. En definitiva, se hace necesario poner en valor el juego real, ya sea en exteriores o en interiores, individual o compartido; y ofrecer a las familias, en la línea de Dardanou et al. (2020), una mayor orientación sobre el uso tecnológico para ofrecer oportunidades y vivencias creativas, sociales y de realización personal en la infancia (Buckingham, 2002; Díaz, 2006). 
Sin duda, la familia se constituye como el principal referente social en estas edades, y parece oportuno pensar que es también quien más influye en este primer momento de conformación del ocio. Sin embargo, no es fácil este rol parental, pues exige reflexión, conciliación, distribución de tiempos y esfuerzo.

Por ello, es importante potenciar el papel de las figuras parentales en la transmisión de un ocio humanista, así como promover la idea de la responsabilidad compartida y el compromiso negociado interfamiliar. Si se logra que la visión familiar sobre el ocio educativo en la infancia sea adecuada, es decir, se tomen decisiones de un ocio personalizado en consonancia con la edad y con el carácter e identidad individual, se estará ayudando a evitar la hiperactividad, el ocio no elegido, consumista y nocivo (Cuenca, 2014).

Para ello, se requiere de orientaciones hacia un ocio activo que ofrezca oportunidades individuales a cada proceso evolutivo y valore los intereses y las motivaciones personales como elementos esenciales para la educación y el desarrollo; un ocio sustancial, como el ocio intergeneracional, dando valor al abuelazgo como clave en la crianza, el cuidado y la educación de las nuevas generaciones, como apuntan investigaciones en esta línea (Alonso et al., 2020); un ocio lúdico o creativo que permita tiempos vacíos de actividades dirigidas, donde exploren el espacio, los materiales e inventen historias, se disfracen y construyan un mundo interno que les permita pensar, imaginar y crear; un ocio cultural y festivo capaz de generar entornos y de ofrecer oportunidades que permitan mostrar la realidad y ampliar el abanico de su cultura; un ocio ambiental-ecológico que fomente la relación y el respeto por la naturaleza; un ocio deportivo que parta del gusto y el disfrute de la experimentación del propio cuerpo, de sus posibilidades y capacidades desde la libertad del movimiento; y un ocio solidario donde se destaquen los valores humanos, potenciando la relación social y el respeto.

En sí mismo, el ocio humanista es un ocio saludable que requiere adaptarse al desarrollo, necesidades, intereses y preferencias en las primeras edades, siendo fundamental para el bienestar y la calidad de vida. Se deben dar las condiciones necesarias para que desde el nacimiento se estimulen esas experiencias de disfrute. Si se logran acumular vivencias satisfactorias en el ámbito del ocio, los resultados perdurarán en otras etapas del desarrollo (Berrios et al., 2017). 


\section{BiBLIOGRAFÍA}

Águila, C. (2007). El ocio y su investigación en los albores del s. XxI: entre la modernidad y la posmodernidad, en M. J. Monteagudo Sánchez (ed.), El ocio en la investigación actual. Documentos de ocio 32. Bilbao: Universidad de Deusto.

Alonso, R. A., Sáenz de Jubera, M. y Sanz, E. (2020). Tiempos compartidos entre abuelos y nietos, tiempos de desarrollo personal. Revista Española de Pedagogía 78, 277. Recuperado de: https://doi.org/10.22550/REP78-32020-01

Andrés, C. y Fernández, A. (2016). Las prácticas de crianza de los padres: su influencia en las nuevas problemáticas en la primera infancia. Revista de Educación Inclusiva 9(1), 30-42.

Aristegui, I. y Silvestre, M. (2012). El ocio como valor en la sociedad actual. ARBOR Ciencia, Pensamiento y Cultura 188(754), 283-291. DOI: https:// doi.org/10.3989/arbor.2012.754n2002

Aucouturier, B. (2018). Actuar, jugar, pensar. Puntos de apoyo para la práctica psicomotriz educativa y terapéutica. Barcelona: Grao.

Azagra, A. y Giménez, V. (2018). El arte en la primera infancia: Propuestas destacables. Revista Sobre La Infancia Y La Adolescencia 0(15), 70-97. DOI: https://doi.org/10.4995/reinad.2018.9600

Barudy, J. y Dantagman, M. (2009). Los buenos tratos a la infancia. Parentalidad, apego y resiliencia. Barcelona: Gedisa.

Berrios, M. A., Lazcano, I. y Madariaga, A. (2017). Intervención en educación del ocio desde un enfoque humanista. Contextos Educativos 2, 131-145. DOI: http://doi.org/10.18172/con.3066

Bofarull, I. (2005). Ocio y tiempo libre: Un reto para la familia (1.a ed., Familia). Navarra: Eunsa.

Braid, C. y Finch, B. (2015). Ah, I know why: children developing understandings through engaging with a picture book. Literacy 49(3), 115-122.

Buckingham, D. (2002). Crecer en la era de los medios electrónicos. Madrid: Morata.

Burdette, H. y Whitaker, R. (2005). Resurrecting Free Play in Young Children. Archives of Pediatrics \& Adolescent Medicine 159(1), 46. DOI: 10.1001/ archpedi.159.1.46 
Caballo, B. (2009). Una aproximación a los tiempos de la infancia, en M. Cuenca Cabeza y E. Aguilar Gutiérrez, El tiempo de ocio: transformaciones y riesgos en la sociedad apresurada. Documentos de estudios de ocio, 36 (pp. 195-217). Bilbao: Universidad de Deusto.

Caballo, M. B., Gradaílle, R. y Merelas, T. (2012). Servicios socioeducativos y corresponsabilidad en la conciliación de los tiempos familiares: Situación de la infancia en la Galicia urbana. Pedagogía Social. Revista Interuniversitaria 20, 179-202.

CAride, J. A. (2014). Del ocio como educación social a la pedagogía del ocio en el desarrollo humano. Edetania: estudios y propuestas socioeducativas (45), 33-54. Recuperado de: http://dialnet.unirioja.es/servlet/ oaiart? codigo $=5010250$

Cartanyà-Hueso, À., Lidón-Moyano, C., Cassanello, P., Díez-Izquierdo, A., Martín-Sánchez, J. C., Balaguer, A. y Martínez-Sánchez, J. M. (2021). Smartphone and tablet usage during COVID-19 pandemic confinement in children under 48 months in Barcelona (Spain). Healthcare (vol. 9, n. ${ }^{\circ}$ 1, p. 96). Multidisciplinary Digital Publishing Institute. Recuperado de: https://doi.org/10.3390/healthcare9010096

Common Sense Inc. (2017). The common sense census: Media use by kids age zero to eight 2017. Common Sense Media. Recuperado de: https://www. commonsensemedia.org/research/the-common-sense-census-media-useby-kids-age-zero-to-eight-2017

Corraliza, J. y Collado, S. (2011). La naturaleza cercana como moderadora del estrés infantil. Psicothema 23(2), 221-226.

Council on Communications and Media (2016). Media and young minds. Pediatrics 138(5), e20162591. DOI: https://doi.org/10.1542/peds.20162591

Cuenca, M. (2004). Pedagogía del Ocio: Modelos y Propuestas. Bilbao. Universidad de Deusto.

Cuenca, M. (2000). Ocio humanista. Dimensiones y manifestaciones actuales del ocio. Bilbao. Universidad de Deusto

Cuenca, M. (2014). Ocio Valioso. Documentos de Estudio de Ocio 52. Bilbao: Universidad de Deusto.

Da Rosa Piccolo, L., Weisleder, A. y Mendelsohn, A. L. (2020). Promoting global early child development through play: two sustainable, effective 
models. Pediatrics 146(6). Recuperado de: https://doi.org/10.1542/ peds.2020-032433

Dardanou, M., Unstad, T., Brito, R., Dias, P., Fotakopoulou, O., Sakata, Y. y O'Connor, J. (2020). Use of touchscreen technology by 0-3-yearold children: Parents' practices and perspectives in Norway, Portugal and Japan. Journal of Early Childhood Literacy 20(3), 551-573. DOI: https:// doi.org/10.1177/1468798420938445

DÁvila, E. (2016). Rápido a ninguna parte. Consideraciones en torno a la aceleración del tiempo social. Acta Sociológica 69, 51-75. Recuperado de: https://doi.org/10.1016/j.acso.2016.02.003

DeClaración de los Derechos del NiÑo (1989). Convención sobre los derechos del niño. Recuperado de: https://www.ohchr.org/sp/professionalinterest/ pages/crc.aspx

De Valenzuela, A., Martínez, R. y Escobar, D. (2021). Prácticas de ocio y tiempo libre de los adolescentes en Galicia: análisis y reflexiones en clave socioeducativa. Bordón. Revista de Pedagogía 73(1), 161-177. DOI: https:// doi.org/10.13042/Bordon.2021.83201

Delgado, I. (2012). Juego infantil y metodología. Madrid: Paraninfo.

DíAz, P. (2006). Efectos del uso de Videojuegos en niños y adolescentes en España y Estados Unidos. El consumo consciente como posible factor reductor de efectos nocivos. Revista de Comunicación y nuevas tecnologías 7, 1-23.

Díaz-Herrera, L., Salcines-Talledo, I. y González-Fernández, N. (2021). El impacto de las tecnologías en el ocio de la primera infancia. Revista Fuentes 23(2). DOI: https://doi.org/10.12795/revistafuentes.2021.12753

Ferreiro-NúÑEz, X. (2016). Animación sociocultural en la infancia: Iniciativas y programas en tiempos de ocio en Galicia. Pedagogía Social 28, 263-266.

Fraguela, R., Lorenzo, J. y Varela, L. (2011). Conciliación y actividad física de ocio en familias con hijos en educación primaria. Implicaciones para la infancia. Revista de Investigación en Educación 9(2), 162-173. Recuperado de: https://revistas.webs.uvigo.es/index.php/reined/article/view/1896

FreIRE, H. (2011). Educar en verde: ideas para acercar a niños y niñas a la naturaleza (1. a ed., Colección Familia y educación 21). Barcelona: Graó.

Gambara, H., Botella, J. y Gempr, R. (2002). Tiempo vacío y tiempo lleno. Un metaanálisis sobre los cambios en la percepción del tiem- 
po en la edad. Estudios de Psicología 23(1), 87-100. DOI: https://doi. org/10.1174/021093902753535204

HAwKEY, E. (2019). Media use in childhood: Evidence-based recommendations for caregivers. CYF News. American Psychological Association. Recuperado de: https://acortar.link/waruh8

Jenssen, B. P., Kelly, M. K., Powell, M., Bouchelle, Z., Mayne, S. L. Y FIKs, A. G. (2021). COVID-19 and changes in child obesity. Pediatrics 147(5). DOI: https://doi.org/10.1542/peds.2021-050123

Kalnina, D. y Kalnins, A. (2020). 2-3-Year-Old Children and the Use of Smart Devices. International Journal of Smart Education and Urban Society (IJSEUS) 11(1), 64-74. Recuperado de: https://doi.org/10.4018/ IJSEUS.2020010105

KhUSNIDAKHON, K. (2021). The importance of enhancing social skills of preschoolers. European Scholar Journal (ESJ) 2(3), 74-78. Recuperado de: https://doi.org/10.47689/2181-1415-vol2-iss2/S-pp583-590

Kotrla Topić, M., Varga, V. y Jelovčić, S. (2021). Digital Technology Use during the COVID-19 Pandemic and Its Relations to Sleep Quality and Life Satisfaction in Children and Parents. Društvena istraživanja: časopis za opća društvena pitanja 30(2), 249-269. DOI: https://doi.org/10.5559/ di.30.2.04

Konok, V., Bunford, N. y Miklósi, Á. (2020). Associations between child mobile use and digital parenting style in Hungarian families. Journal of Children and Media 14(1), 91-109. DOI: https://doi.org/10.1080/1748279 8.2019.1684332

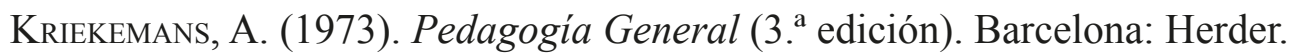
LeCuyer, C. (2012). Educar en el asombro. Barcelona: Plataforma.

LeYrA, B. y BÁrcenAs, A. M. (2014). Reflexiones etnográficas sobre el ocio infantil. Revista Latinoamericana de Metodología de las Ciencias Sociales 4(1). Recuperado de: http://sedici.unlp.edu.ar/handle/10915/38187

Limone, P. у Tото, G. A. (2021). Psychological and emotional effects of Digital Technology on Children in Covid-19 Pandemic. Brain Sciences 11(9), 1126. Recuperado de: https://doi.org/10.3390/brainsci11091126

Louv, R. (2008). Last child in the woods: Saving our children from nature-deficit disorder. NC Algonquin Books. 
Marín-Díaz, V. y SÁnchez-Cuenca, C. (2015). Formación en valores y cuentos tradicionales en la etapa de educación infantil. Revista Latinoamericana de Ciencias Sociales, Niñez y Juventud 13(2), 1093-1106. DOI: https://doi. org/10.11600/1692715x.13238190514.

Marín, M. del P., Quintero, P. A. y Rivera, S. C. (enero-junio, 2019). Influencia de las relaciones familiares en la primera infancia. Poiésis(36), 164-183. DOI: https://doi.org/10.21501/16920945.3196

McCABE, S. (2015). Family leisure, opening a window on the meaning of family. Annals of Leisure Research 18(2), 175-179. DOI: https://doi.org/1 0.1080/11745398.2015.1063748

Mora, F. (2013). Neuroeducación: Solo se puede aprender aquello que se ama . Madrid: Alianza Editorial.

MorÁn de CAStro, M. C. (2009). Infancia y familias a ritmo del tiempo escolar, en M. Cuenca Cabeza y E. Aguilar Gutierrez (eds.), El tiempo del ocio: transformaciones y riesgos en la sociedad apresurada. Documentos de Estudios de Ocio 36, 177-193. Barcelona: Graó.

ONu (2015). Transformar nuestro mundo: la Agenda 2030 para el Desarrollo Sostenible. Recuperado de: https://acortar.link/CzXt61

Palacios, J. y Castañeda, E. (2009). Principios y retos de la educacion inicial que queremos para la generación de los Bicentenarios, en J. Palacios, E. Castañeda y Fundación Santillana (coords.), La primera infancia (0-6 años) y su futuro (pp. 115-124). Madrid: Organización de Estados Iberoamericanos para la Educación, la Ciencia y la Cultura (OEI). Fundación Santillana.

Piaget, J. (2000). El nacimiento de la inteligencia del niño. Barcelona: Crítica. PIKLER, E. (1984). Moverse en libertad: Desarrollo de la motricidad global (Educación Hoy). Madrid: Narcea.

Romero, V. y Gómez, M. (2016). El juego infantil y su metodología. Barcelona: Altamar.

Rodrigo, M. J. (2015). Preservación familiar y parentalidad positiva: dos enfoques en convergencia. Revista de Treball Social de Catalunya 204, 3647. Recuperado de: https://tiab-badalona.cat/wp-content/uploads/2018/12/ parentalidad-positiva-art-rts.pdf

Rodrigo-Moriche, M. P. y VAllejo, S. (2018). Nuevos horizontes de ocio y participación infantil: construyendo ciudadanía desde los intereses y necesidades de los niños, niñas y adolescentes (NNA), en R. A. Alonso, M. 
Sáenz de Jubera Ocón y E. Sanz Arazuri (2020), Tiempos compartidos entre abuelos y nietos, tiempos de desarrollo personal. Revista de Pedagogía 78(277), 415-433.

SANZ, M. J. (2014). Las necesidades de la primera infancia. Los calendarios y guías del desarrollo como instrumentos de apoyo para la crianza. Seminario de intervención y políticas sociales (SIPOSO).

Sanz Arazuri, E., SÁenz de Jubera Ocón, M. y Cano, R. (2018). Actitudes de padres e hijos hacia un ocio compartido en familia. Pedagogía Social revista Interuniversitaria 32, 59-70. Recuperado de: https://doi.org/10.7179/ PSRI_2018.32.05

Sarrate, M. L. (2008). Ocio y tiempo libre en los centros educativos. Bordón 60, 51-61. Recuperado de: Dialnet-OcioYTiempoLibreEnLosCentrosEducativos-2912271 (4).pdf

Skora, E. y Poenlmann-Tynan, J. (2020). In-home video chat for young children and their incarcerated parents. Journal of Children and Media 14(3), 400-406. DOI: https://doi.org/10.1080/17482798.2020.1792082

Stebirns, R. (2012). Compromiso temporal discrecional: efectos sobre la elección y el estilo de ocio. Arbor 188, 293-300. DOI: http://doi.org/10.3989/ arbor.2012.754n2003

Strouse, G. A., McClure, E., Myers, L. J., Zosh, J. M., Troseth, G. L., BlanchFIELD, O. ..., y BARR, R. (2021). Zooming through development: Using video chat to support family connections. Human Behavior and Emerging Technologies. Recuperado de: https://doi.org/10.1002/hbe2.268

Unicef (2014). Vulnerabilidad y exclusión en la infancia. Análisis de los factores de exclusión social: Propuesta de un sistema de información sobre la infancia en exclusión. Madrid. Recuperado de: https://acortar.link/R9cgns UnICEF (2018). Informe anual para cada niño, todos los derechos. Madrid: Universidad de Navarra.

Valdemoros, M. A., Ponce de León, A., Sanz-Arazuri, E. y Caride, J. A. (2014). La influencia de la familia en el ocio físico-deportivo juvenil: nuevas perspectivas para la reflexión y la acción. Arbor 770, 190-92. Recuperado de: http://dx.doi. org/10.3989/arbor.2014.770n6013

Wang, G., Zhang, Y., ZhaO, J., Zhang, J. y JiAnG, F. (2020). Mitigate the effects of home confinement on children during the COVID-19 outbreak. Lancet 395, 945-947. DOI: https://doi.org/10.1016/S0140-6736(20)30547-X 
Weiland, C., Greenberg, E., Bassok, D., Markowitz, A., Rosada, P. G., LuetMER, G., ..., y SNOw, C. (2021). Historic crisis, historic opportunity. Using Evidence to Mitigate the Effects of the COVID-19 Crisis on Young Children and Early Care and Education Programs. University of Michigan and Urban Institute, 41 p. Recuperado de: https://acortar.link/evT1g2

WeLLs, N. M. (2000). At home with nature: Effects of "greenness" on children's cognitive functioning. Environment and behavior 32(6), 775-795.

WLRA (1994). International Charter for Leisure Education. Education, World Leisure \& Recreation 36(2), 41-45. DOI: https://doi.org/10.1080/1026113 3.1994.9673916

World Health Organization (2019). Guidelines on physical activity, sedentary behaviour and sleep for children under 5 years of age. World Health Organization. Recuperado de: https://acortar.link/tFxPUA

Yogman, M., Garner, A., Hutchinson, J., Hirsh-Pasek, K., Golinkoff, R. M. y Committee on Psychosocial Aspects of Child and Family Health (2018). The power of play: A pediatric role in enhancing development in young children. Pediatrics 142(3), e20182058pmid:30126932. DOI: https:// doi.org/10.1542/peds.2018-2058

Zuhro, D. F. (2021). Efforts To Stabilize Children's Mental Health Through Screening The Use Of Gadgets In Children In The Pandemic Period Of Covid-19. Journal of Public Health Science Research 1(2), 39-42. Recuperado de: http://journal.umg.ac.id/index.php/jphsr/article/view/2492 